\title{
Advanced analysis of marine plankton using flow cytometry
}

Matthew Haynes, Brian Seegers, and Alan Saluk

Flow Cytometry Core Facility, The Scripps Research Institute, La Jolla, CA

In collaboration with ACEA Biosciences, Inc., San Diego, CA

Flow cytometry has routinely been used to study phytoplankton by taking advantage of innate fluorescence molecules that distinguish different species and their physiological conditions. Recent advances in cytometer design and functionality have modernized certain aspects of marine biology applications creating a more accurate, data-rich, and timely assessment of microscopic marine organisms. We will examine the distribution and abundance of the phytoplankton, bacterial, and viral fractions of seawater determined using the two elements of the ACEA Biosciences Novocyte ${ }^{\mathrm{TM}}$ benchtop flow cytometer; volumetric sample delivery via precise syringe injection and extended fluorescence profiling with three laser excitation.

\section{Sample Collection}

Samples were analyzed from the Scripps Institution of Oceanography Pier in La Jolla, CA. Field samples were collected in sterile tubes and fixed with $0.02 \mu \mathrm{m}$ filtered glutaraldehyde $\left(0.5 \%\right.$ final concentration, incubated at $4^{\circ} \mathrm{C}$ for 20 minutes), flash frozen in liquid nitrogen, and stored at $-80^{\circ} \mathrm{C}$ (Brussard, 2008). Samples were thawed in small batches in a $20^{\circ} \mathrm{C}$ water bath. Unstained samples were analyzed immediately for photosynthetic plankton groups. Heterotrophic bacterial and viral populations are stained using the nucleic acid stain SYBR® Green I (1:10,000 final concentration, incubate at room temp for 15 minutes).

\begin{tabular}{|c|c|c|c|}
\hline Pigment & Channel & Excitation & Emission \\
\hline Chlorophyll & BL4 & 488 & $675 / 30$ \\
\hline Phycoerythrin & BL2 & 488 & $585 / 40$ \\
\hline Phycocyanin & RL1 & 640 & $675 / 30$ \\
\hline Divinyl Chlorophyll a & VL5 & 405 & $675 / 30$ \\
\hline SYBR Green & BL1 & 488 & $530 / 30$ \\
\hline Light Scatter & FSC/SSC & 488 & $488 / 10$ \\
\hline
\end{tabular}

Eukaryotes (Euk01-05) - High chlorophyll, large cells, some classes phycoerytherin positive

Cryptophytes (Crypto) - High chlorophyll, large cells, allophycocyanin positive

Pico Eukaryotes (PicoEuk) - Low chlorophyll, smaller cells, no phycoerytherin

Synechococcus PE+ (PE+Euk) - Low chlorophyll, small cells, phycoerytherin positive

Synechococcus APC+ (Syn-PC+) - Low chlorophyll, small cells, allophycocyanin positive

Prochlorococcus (Pro) - Low chlorophyll, small cells, divinyl chlorophyll-a positive

\section{Sample Analysis}

Photosynthetic pico(<2um) and nano(2-20um) plankton groups (eukaryotic and prokaryotic) were assessed using natural pigment fluorescence and scatter. Signal detection is triggered on chlorophyll fluorescence, using $0.2 u m$ filtered seawater to set threshold. Heterotrophic bacterial and viral populations were distinguished using the nucleic acid stain SYBR Green I. Signal detection is triggered on nucleic acid fluorescence, again using 0.2um filtered seawater to set threshold.

\section{Summary}

The use of the multi-laser format $(405,488$, and $640 \mathrm{~nm}$ excitations) allows for reliable discrimination of phototrophic eukaryotes, Synechococcus spp., Prochlorococcus spp., and heterotrophic populations in aquatic samples. Precision syringe driven fluidics enables direct enumeration of populations allowing for reliable comparisons to assess population dynamics. The integrated NovoSampler ${ }^{\mathrm{TM}}$ permits timely, automatic processing of samples in a variety of tube or plate formats that can be configured by the user. Fixed PMT voltages facilitates quick setup and experimental repeatability, while requiring less user training. The system's innovative engineering allows for compact design with a small bench top footprint and low power demand making it ideal for shipboard analysis of marine samples eliminating the need for research samples to be stored and analyzed at a later date.

To read more, visit Aceabio.com.
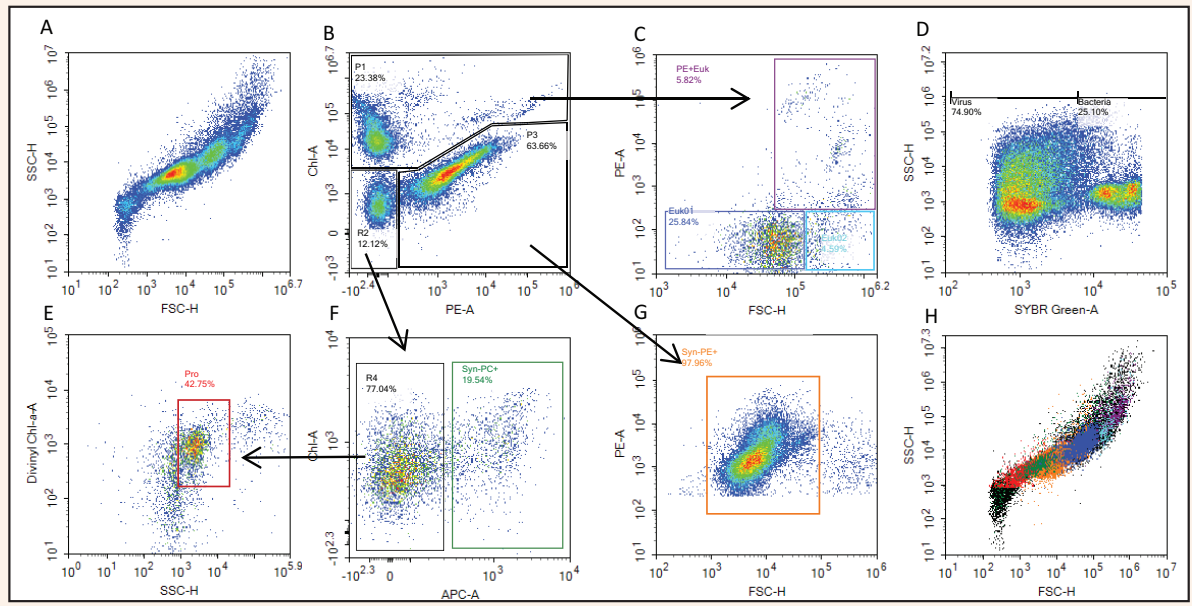

Figure 1. Flow cytometric analysis of plankton from the Scripps Institute of Oceanography pier. A-G) Density plots with gating strategies. Six photosynthetic groups were identified, three eukaryotic (C) and 3 cyanobacteria (E,F,G). Synechococcus PE+ dominate group $(B, G)$. D) Bacteria were distinguished from viral populations using SYBR green. H) Dot plot of forward and side scatter, identifying phytoplankton groups by color. 